\title{
Placental Thickness as a Sonological Parameter for Estimating Gestational Age
}

Paul Jacob¹, Pranesh Panneerselvam², Nasin Usman³, Bharathi Vivekanandan, Padmapriya Chandrakumar ${ }^{5}$

1Junior Resident, Department of Radiodiagnosis and Imaging Sciences, Sri Ramachandra Institute of Higher Education and Research, Chennai, Tamilnadu, India. ${ }^{2}$ Senior Resident, Department of Radiodiagnosis and Imaging Sciences, Sri Ramachandra Institute of Higher Education and Research, Chennai, Tamilnadu, India. 3Junior Resident, Department of

Radiodiagnosis and Imaging Sciences, Sri Ramachandra Institute of Higher Education and Research, Chennai, Tamilnadu, India. ${ }^{4}$ Junior Resident, Department of Radiodiagnosis and Imaging Sciences, Sri Ramachandra Institute of

Higher Education and Research, Chennai, Tamilnadu, India. ${ }^{5}$ Senior Resident, Department of Radiodiagnosis and Imaging Sciences, Sri Ramachandra Institute of Higher Education and Research, Chennai, Tamilnadu, India.

\section{ABSTRACT}

\section{BACKGROUND}

Placental thickness can be used to estimate the gestational age; however, total placental volume is probably the most accurate estimate of placental size, but volumetric measurement is too complicated and cumbersome for routine use, whereas the measurement of placental thickness is relatively simple and clinically useful. We wanted to evaluate the placental thickness as a parameter for estimating gestational age of the foetus \& assess the growth pattern of placenta with advancing gestational age.

\section{METHODS}

Women referred for routine antenatal ultrasound from 11 to 40 weeks of gestational age were included in the study. Patients with PIH, diabetes mellitus, poly/oligohydramnios, IUGR, hydrops foetalis, congenital malformations, multifoetal pregnancy and with variations in insertions of umbilical cord like marginal or battledore placentas and velamentous insertions are excluded from the study. Grey scale real time ultrasonographic examinations were performed using 3.5 $\mathrm{MHz}$ convex array transducer. To obtain an accurate placental measurement, it is important to identify the placental-myometrial interface, when placenta is posterior, identification of this region is facilitated by the acquisition of images as free from acoustic shadowing from the foetus as possible.

\section{RESULTS}

It was observed that the placental thickness gradually increased from approximately $11.4 \mathrm{~mm}$ at 11 weeks to $36.5 \mathrm{~mm}$ at 40 weeks of gestation. From 11 to 35 weeks of gestation, the placental thickness (in $\mathrm{mm}$ ) almost matched the gestational age in weeks, thereafter from 36 to 40 weeks; the placental thickness was lower by $1-3 \mathrm{~mm}$.

\section{CONCLUSIONS}

The relationship between the placental thickness and gestational age is linear and direct. Placental thickness (in $\mathrm{mm}$ ) measurement can be used as an important additional parameter for estimating gestational age along with other parameters especially from 11 to 35 weeks of gestation. Accuracy of placental measurements depends on making a perpendicular scan of the placenta and care should be taken in acquisition and interpretation of images to prevent spurious measurements.

\section{KEY WORDS}

Placental Thickness, Sonological Parameters, Gestational Age, Antenatal Ultrasound
Corresponding Author: Pranesh Panneerselvam, No. 1. Ramachandra Nagar, Porur, Chennai, Tamilnadu, India.

E-mail: drpranesh19@gmail.com

DOI: $10.14260 / j e m d s / 2019 / 668$

Financial or Other Competing Interests: None.

How to Cite This Article:

Jacob P, Panneerselvam P, Usman N, et al. Placental thickness as a sonological parameter for estimating gestational age. J. Evolution Med. Dent. Sci. 2019;8(41): 3074-3079, DOI:
Submission 13-08-2019, Peer Review 27-09-2019, Acceptance 04-10-2019, Published 14-10-2019. 


\section{BACKGROUND}

Placenta is a foetal organ with important metabolic, endocrine and immunological functions. It also has a role in protecting the foetus from noxious agents. Placental evaluation by ultrasonography has been used to characterize placental position and morphologic changes as the placenta matures, one additional ultrasonographic parameter frequently used to assess the placenta is placental size. ${ }^{1}$ Currently the most effective way to date pregnancy is by the use of ultrasound. Placental thickness can be used as a new parameter to estimate the gestational age however total placental volume is probably the most accurate estimate of placental size, but volumetric measurement is too complicated and cumbersome for routine use, whereas the measurement of placental thickness is relatively simple and clinically useful. The measurement of placental thickness is relatively simple and clinically useful. Abnormal thickness of placenta is well recognized as a diagnostic harbinger in a wide spectrum of pathologic events. Placental thickness can contribute to the management of foetus at risk. ${ }^{2} \mathrm{Few}$ authors have studied the role of placental thickness as a new parameter for estimating gestational age and placental thickness nomograms in relation to gestational age have been published. Placental thickness measurement can differentiate normal from abnormal pregnancy. ${ }^{3}$ Accurate assessment of gestational age is an important part of any obstetric examination and presently the most effective way to date pregnancy is by the use of ultrasound. Several sonographically derived foetal parameters are used to date pregnancy. They are foetal crown-rump length (CRL), biparietal diameter (BPD), head circumference (HC), femur length (FL) and abdominal circumference (AC). Placental thickness measurement can be used as a new parameter to estimate gestational age. Single most useful piece of information that obstetric sonography provides is the accurate determination of menstrual age. ${ }^{4}$ Knowledge of menstrual age is important to obstetrician because it affects clinical management in a number of important ways.

\section{Placental Maturation}

A sonographic classification system for grading placentas in utero according to maturational changes has been proposed by Grannum and associates. ${ }^{5}$ Grade 0: The placental tissue and the basal plate are homogeneous without the presence of linear highly reflective foci. The chorionic plate is smooth and well defined. Grade 1: The placental tissue contains a few linear highly reflective foci parallel to the basal plate, which remains unchanged. The chorionic plate presents subtle undulations. Grade 2: The placental tissue contains randomly dispersed echoes and is divided by comma like reflective structures continuous with the chorionic plate. The marked indentations of the chorionic plate do not reach basal plate, which is well defined by small linear highly reflective areas. Grade 3: The placental tissue is divided into compartments containing central echo free areas. The chorionic plate indentations reach the basal plate, which contains almost confluent, very highly reflective areas.

\section{Delayed/Accelerated Maturation}

Placental maturity may be accelerated or delayed in complicated pregnancy. In making the diagnosis one must be certain of the gestational age. Accelerated maturation is identified by finding abnormally small villi, an abnormally thin syncytiotrophoblastic cell layer covering the villi. When present, accelerated maturation can be relatively uniform throughout the placenta, or it can be interspersed with areas that appear normally mature for gestational age.

\section{Uniformly Accelerated Maturation}

The antecedents are being absence of maternal thirdtrimester peripheral edema, low maternal net pregnancy weight gain, and low maternal pregravid weight for height. The only unfavourable outcome was for stillbirths.

\section{Unevenly Accelerated Maturation}

An uneven acceleration of placental maturation is widely recognized as a manifestation of stenoses and occlusions in the uterine spiral arteries that unevenly reduce blood flow to the intervillous space in the placenta. This uneven blood flow, when present for several weeks or longer, accelerates villous maturation in those areas of the placenta where blood flow into the intervillous space is low, while acceleration is absent in the areas where the blood flow to intervillous space is normal. The cause of uneven acceleration presumed to be a combination of fluctuating vasoconstriction and longstanding stenotic lesions. Placental infarcts are a common associated finding. Risk factors include pre-eclampsia, chronic hypertension, intrauterine growth retardation and eclampsia. ${ }^{6}$ Others include white race, primigravida, low maternal pre-pregnancy weight gain, and overweight mother for height before pregnancy. Stillbirths and neonatal deaths are increased.

\section{Delayed Maturation of the Placenta}

Delayed placental maturation is less frequent than accelerated maturation. The antecedents are maternal diabetes mellitus, major foetal malformations, and erythroblastosis foetalis. Delayed placental maturation is associated with an increased risk of stillbirths, neonatal deaths, and mental retardation.

\section{Focal Cystic/Hypoechoic Lesions}

Cystic or hypo echoic lesions are ubiquitous in placenta after 25 weeks. They represent a variety of entities, including intervillous thrombosis, subchorionic fibrin deposition, perivillous fibrin and decidual septal cyst.

Lesions Resulting from Maternal Blood Flow Disturbances

1. Massive perivillous fibrin deposition: These are located in the peripheral area or marginal angle of placenta. These deposits occur in nearly all full-term pregnancy, but macroscopically visible plaques occur in $20 \%$ to $25 \%$ of uncomplicated pregnancies.

2. Subchorionic fibrin deposition: These are triangular or rectangular areas of fibrin deposited under the chorion or foetal surface of placenta. They are noted in approximately $20 \%$ of placentas and are not associated with any maternal factors. They appear anechoic on ultrasound and do not show flow on colour Doppler.

3. Intervillous thrombi: These are usually $1-2 \mathrm{cms}$ in diameter and consist of coagulated maternal blood in the intervillous space. These are very common and appear 
hypoechoic on ultrasound. Intervillous thrombi have no effect on placental function or foetal health.

4. Placental Infarction: A placental infarct is defined as an area of ischemic villous necrosis. Infarcts are usually the result of the occlusion of one or more spiral arteries in the uterine wall. Such occlusions are common with disorders that unevenly reduce uteroplacental blood flow. The most common of these are preeclampsia, eclampsia, and chronic maternal hypertension. One or two small infarcts, and even larger infarcts that are at the margin of the placenta are not usually associated with unfavorable pregnancy outcomes in full-term infants. As the number and size of infarcts increase, so do the frequencies of stillbirth and neonatal death. Overall the perinatal mortality rate associated with placental infarction increases with the size of the infarct, with preterm delivery, and with the presence of disorders that reduce placental function such as preeclampsia, eclampsia, chronic maternal hypertension, and lupus erythematosus. One to four grossly visible infarcts and infarcts $>3 \mathrm{~cm}$ in diameter are associated with increased risk for foetal growth retardation.

5. Maternal Floor Infarction: Also called massive basal plate fibrin deposition. The fibrin is deposited in the basal plate from maternal blood in the intervillous space. It is very rare, occurring in $0.1 \%-0.5 \%$ of pregnancies. This lesion may interfere with the perfusion of the intervillous space by maternal blood and is associated with a high incidence of foetal death or IUGR. On ultrasound, typical basal location near the decidua is characteristic.

\section{Growth Pattern of Normal Placenta}

Growth of placenta results from multiplication and branching of chorionic villi. ${ }^{7}$ Growth can be estimated by measuring the thickness or by an estimation of placental volume. Placenta grows throughout pregnancy, initial growth being much more rapid than that of the foetus. ${ }^{8}$ Placental and foetal weights are closely correlated in most circumstances and it follows nearly a linear pattern except during last few weeks of gestation. Placenta having reached a size sufficient to meet its transfer function adequately grows little nearer term ${ }^{9}$ and the ratio of foetal weight to placental weight increases towards term. ${ }^{10}$ Grannum et al reported that placental thickness would increase linearly until 33 weeks of pregnancy, after which there was gradual thinning. ${ }^{5}$ Other authors reported similar findings. Berkowitz et al reported gradual decrease in placental size after 32 weeks until term. Bleker et al postulated that placental volume decrease towards term might be due to a decrease of blood volume of intervillous space. They also found that human placenta stops growing before the end of pregnancy. ${ }^{11}$ Jauniax et al also reported reduced placental volume growth rate after 30 weeks. ${ }^{12}$

\section{METHODS}

Prospective cross-sectional study was conducted in 200 antenatal women of all gestational ages from 11 weeks to 40 weeks of gestation referred for routine antenatal ultrasound. Patients with PIH, Diabetes mellitus, Poly/Oligohydramnios, IUGR, hydrops foetalis, congenital malformations, Multifetal pregnancy and with variations in insertions of umbilical cord like marginal or battledore placentas and velamentous insertions are excluded from the study.

Gray scale real time ultrasonographic examinations were performed using $3.5 \mathrm{MHz}$ convex array transducer. The placental thickness in $\mathrm{mm}$ was measured at cord insertion site with transducer oriented perpendicular to both the chorionic and basal plates. Cord insertion site is usually central but slightly eccentric position may be normal. The ultrasonic appearance of the cord insertion appears either as hypoechoic areas closest to the chorionic plate with a v shape or as linear echoes emanating at right angles from the placental surface, placental thickness was calculated from the echogenic chorionic plate to placental myometrial interface.

The gestational age in first trimester from 11 to 13 weeks of pregnancy was determined by measuring CRL and calculations using Hadlock tables additional measurements are not more accurate than the CRL length in predicting age from 11 to 13 weeks and their use in conjunction with CRL does not further improve age estimation.13 The gestational age in second and third trimesters from 14 to 40 weeks of pregnancy was determined by composite foetal measurements of BPD, HC, AC, and FL gestational age was computed by the ultrasound machine based on Hadlock tables by using regression equations from combination of measurements (computation software package).

Patients were then categorized into two groups based on placental location. $(2,3,4)$ Group I: Anterior and lateral placentas combined. (Figure 1 and 3). Group II: Posterior and fundal placentas combined. (Figure 2 and 4 )

Correlation of mean placental thickness with calculated gestational age from 11 weeks to 40 weeks was obtained in each group separately.

\section{RESULTS}

\begin{tabular}{|c|c|c|}
\hline Age in Years & Number Patients & Percentage \\
\hline$<20$ & 15 & $7.5 \%$ \\
\hline $20-25$ & 93 & $46.5 \%$ \\
\hline $25-30$ & 80 & $40.0 \%$ \\
\hline$>30$ & 12 & $6.0 \%$ \\
\hline \multicolumn{3}{|c|}{ Maternal Age Distribution } \\
\hline Most of the antenatal women included in the study are in the age group of $20-25$
\end{tabular}
yrs.

\begin{tabular}{|c|c|c|}
\hline Placental Position & Number Patients & Percentage \\
\hline Anterior & 72 & $36.0 \%$ \\
\hline Posterior & 45 & $22.5 \%$ \\
\hline Fundal & 48 & $24.0 \%$ \\
\hline Lateral & 35 & $17.5 \%$ \\
\hline \multicolumn{2}{|c|}{ Distribution of Placental Position } \\
\hline $\begin{array}{l}\text { In our study, anterior location of the placenta is found in 36\% of the antenatal } \\
\text { cases, in 24\% of the cases in fundal position in 22.5\% \& 17.5\% of the cases in } \\
\text { posterior \& lateral position respectively. }\end{array}$ \\
\hline
\end{tabular}

\section{Statistical Analysis}

The data collected in this study is analysed statistically by computing the descriptive statistics viz., mean, and SD \& 95\% Confidence Interval were also calculated. The correlation between gestational age \& placenta thickness and gestational is computed. The results are considered statistically significant whenever $\mathrm{p} \leq 0.05$. 
The mean placental thickness with standard deviation and $95 \%$ confidence interval for each gestational age is calculated.

\begin{tabular}{|c|c|c|c|c|c|}
\hline & Range & Mean & $\begin{array}{c}\text { SD } \\
\text { t- Value }\end{array}$ & $\begin{array}{c}\text { Pearson } \\
\text { Correlation }\end{array}$ & $\begin{array}{c}\text { p- } \\
\text { Value }\end{array}$ \\
\cline { 1 - 4 } $\begin{array}{c}\text { Gestational Age } \\
\text { (weeks) by }\end{array}$ & $11-35$ & 24.67 & 7.67 & & \\
\hline USG & & & 0.100 & 0.921 & $<0.001$ \\
\hline Placental & $11-37$ & 24.59 & 7.41 & & \\
\cline { 1 - 2 } Thickness (mm) & & & & \\
\hline \multicolumn{7}{|c|}{ Correlation between 11-35 Weeks of Gestational Age and } \\
Placental Thickness (mm)
\end{tabular}

At 11-35 weeks of gestational age there is no significant difference in mean between the gestational age $(24.67 \pm 7.67)$ and placental thickness $(24.59 \pm 7.41)$ High degree of positive correlation between gestational age and placenta al thickness( $\mathrm{r}=0.921)$ which is significant $(\mathrm{p}<0.001)$.

\begin{tabular}{|c|c|c|c|c|c|}
\hline & Range & Mean & SD t-Value & Pearson Correlation & p \\
\hline Gestational & $36-40$ & 37.30 & .15 & & \\
\hline Age & & & & & \\
\hline (weeks) by & & & & & \\
\hline USG & & & 8.242 & 0.17 & $>0.235$ \\
\hline Placental & $31-38$ & 35.02 & 1.53 & & \\
\hline thickness & & & & & \\
\hline (mm) & & & & & \\
\hline \multicolumn{7}{|c|}{ Correlation between 36-40 Weeks of Gestational Age and } \\
Placental Thickness (MM) \\
\hline
\end{tabular}

After 35 weeks of gestational age there is a high difference in mean between gestational age (37.30 \pm 1.15$)$ and placental thickness $(35.02 \pm 1.53)$. Poor positive correlation between gestational age and placental thickness( $r=0.17$, $\mathrm{p}>0.235$ ) which is statistically not significant.

\begin{tabular}{|c|c|c|}
\hline & $\begin{array}{c}\text { Mean Placental } \\
\text { Thickness }\end{array}$ & $\begin{array}{c}\text { Pearson Correlation with } \\
\text { Gestational Age }\end{array}$ \\
\hline Group 1 & 24.98 & $\mathrm{r}=0.9931$ \\
\hline Group 2 & 25.36 & $\mathrm{r}=0.9919$ \\
\hline \multicolumn{2}{|c|}{ Relationship between Gestational Age and Placental Thickness for } \\
different Placental Locations
\end{tabular}

The relationship between placental thickness and gestational age in both the groups are similar in terms of Pearson correlation co-efficient $(r=0.9931$ for group $1 \& r=$ 0.9919 for group II) Thickness of the placenta did not vary relative to the placental location.

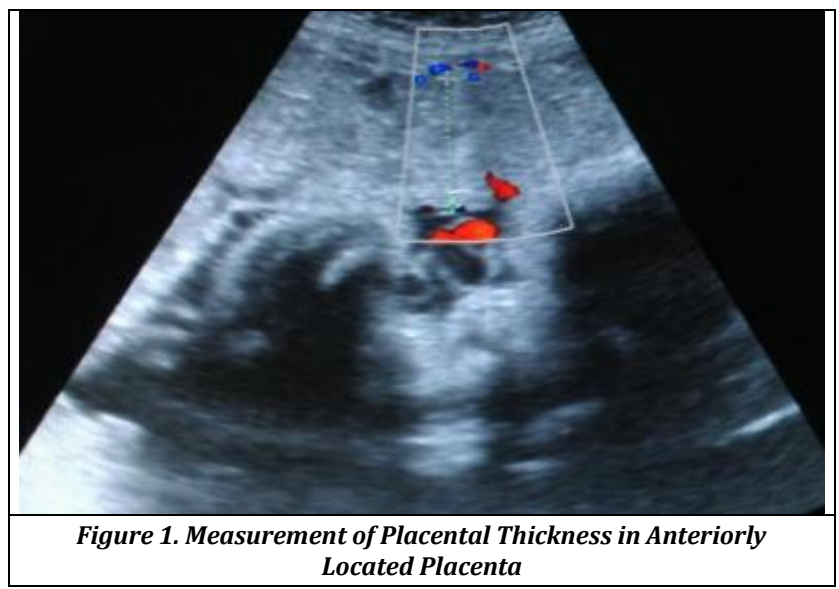

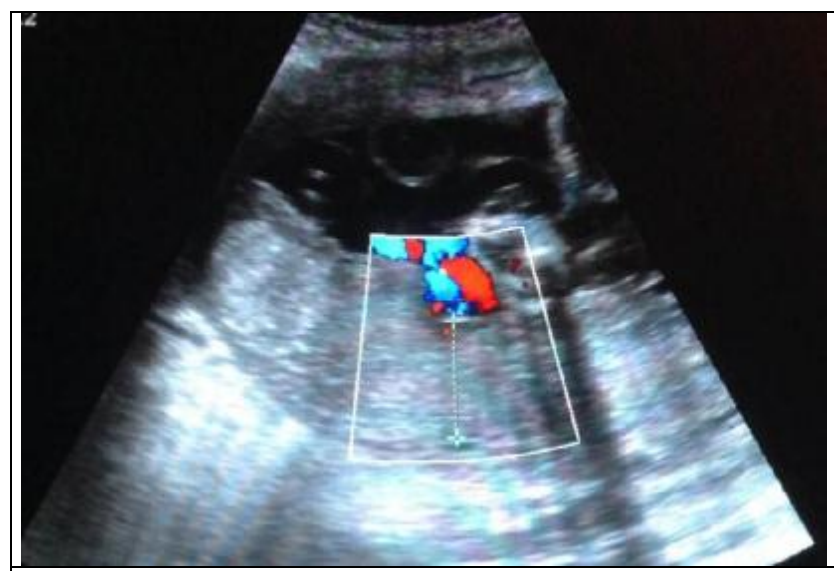

Figure 2. Measurement of Placental Thickness in Posteriorly Located Placenta

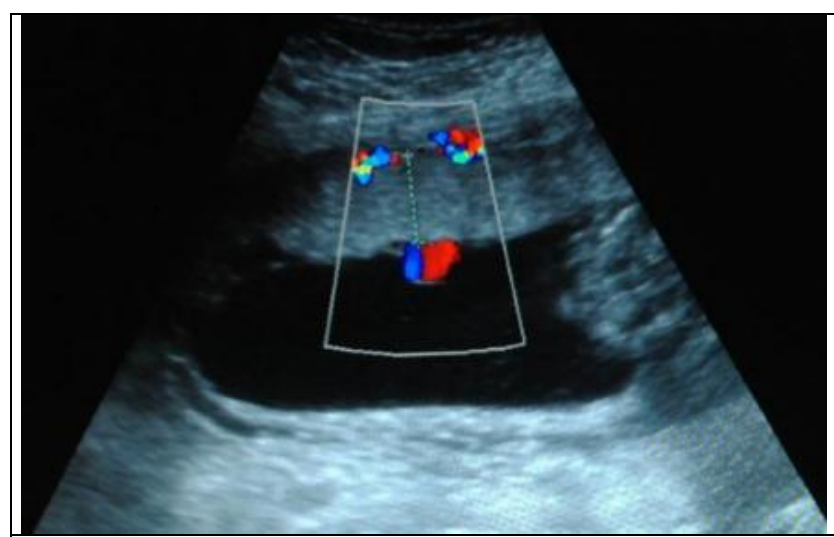

Figure 3. Measurement of Placental Thickness in a Placenta at Lateral Position

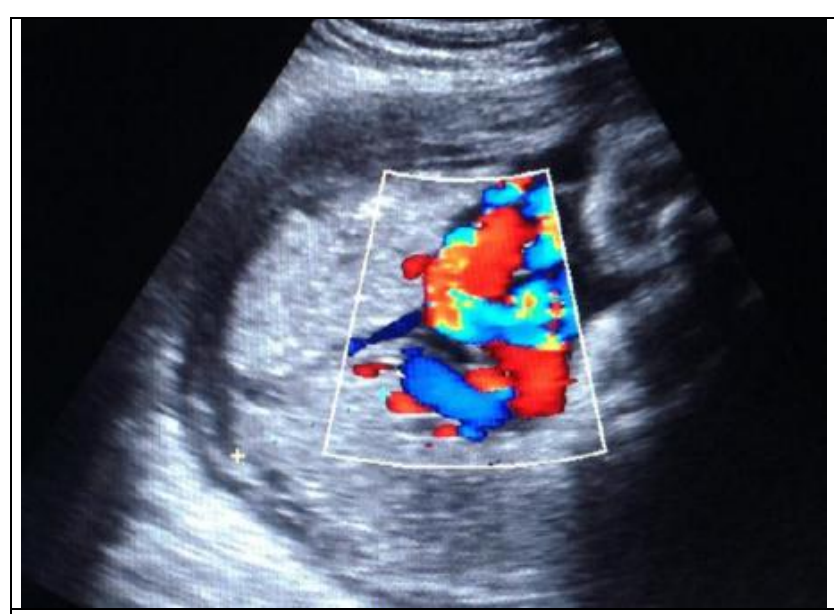

Figure 4. Measurement of Placental Thickness in a Placenta at Fundal Position

\section{DISCUSSION}

For many years sonologists have approached the placenta as a 'static' feature in a dynamic system. ${ }^{5}$ All measurements of foetus were related to menstrual age, the placental thickness was judged as normal or abnormal based on a single "cut off "point whereas the present study data confirm that placental thickness is a function of age. To determine a given placental thickness is normal or abnormal, normal placental thickness must be defined for each week of gestational age throughout pregnancy. 
Abnormal thickening or thinning must be correlated with other estimates of pregnancy duration, the present study assessed the relationship of placental thickness (in $\mathrm{mm}$ ) with sonographic gestational age (in weeks), study showed that the placental thickness (in $\mathrm{mm}$ ) increases steadily with increasing gestational age (in weeks) in a linear fashion and almost matching the gestational age from 11-35 weeks of gestation, the rate of increase of placental thickness gradually diminished from 36-40 weeks and was less by 1-4 mm compared to gestational age (in weeks). ${ }^{14}$

Mital P and Hooja $\mathrm{N}$ also found an increasing trend in the values of mean placental thickness (in $\mathrm{mm}$ ) with increase in gestational age (in weeks) and the placental thickness (in $\mathrm{mm}$ ) coincides almost exactly with the gestational age in weeks. ${ }^{15}$ Anupama Jain et al reported similar correlations between placental thickness and gestational age. They found placental thickness (in mm) almost matched gestational age (in weeks) from 27 weeks to 33 weeks of gestation.16 Grannum et al reported that placental thickness would increase linearly until 33 weeks of pregnancy, after which there was gradual thinning. ${ }^{5}$ Other authors reported similar findings. Berkowitz et al reported gradual decrease in placental size after 32 weeks until term. C.C. Ohagwu, P.O. $\mathrm{Abu}$ et al reported that placental thickness has a strong positive correlation with BPD and AC; with both parameters having identical relationship with placental thickness. ${ }^{17}$

The rate of growth of placental thickness did not vary relative to the placental location as observed in the present study. The growth patterns of anterior and lateral placenta were similar to posterior and fundal placentas as seen in the present study. Similar observations were made by Hoddick et al (1985).18

Certain diseases or abnormalities of the foetus can be detected through measurement of placental thickness. Thin placenta is often a marker for a small for date foetus and a sign of growth restriction. It is also seen in patients with pre eclampsia, chromosomal abnormalities and severe intrauterine infection. Thick placentas are associated with hydrops foetalis, diabetes mellitus and intrauterine infections. It is also associated with increased perinatal risk with increased mortality related to foetal anomalies.

To obtain an accurate placental measurement, it's important to identify the placental-myometrial interface, when placenta is posterior, identification of this region is facilitated by the acquisition of images as free from acoustic shadowing from the foetus as possible. When the placenta is anterior, proper transducer position and gain settings are important to minimize near field and reverberation artefacts correct identification of the placental-myometrial interface should also preclude the illusion of placental thickening induced by focal myometrial thickening.

\section{Limitations}

Accuracy of placental measurements depends on making a perpendicular scan of the placenta and care should be taken in acquisition and interpretation of images to prevent spurious measurements. A method to estimate the thickness of the in-situ placenta from ultrasound images in a single dimension has its own limitations. Cord insertion site on the placenta was difficult to image in normal term pregnancies, especially in posterior locations. 11

\section{CONCLUSIONS}

The relationship between the placental thickness and gestational age is linear and direct. Placental thickness (in $\mathrm{mm}$ ) measurement can be used as an important additional parameter for estimating gestational age along with other parameters especially from 11 to 35 weeks of gestation. Accuracy of placental measurements depends on making a perpendicular scan of the placenta and care should be taken in acquisition and interpretation of images to prevent spurious measurements.

\section{REFERENCES}

[1] Tongsong T, Boonyanurak P. Placental thickness in the first half of pregnancy. J Clin Ultrasound 2004;32(5):231-4.

[2] Kobayashi M, Hellman LM, Fillisti L. Placental localization by ultrasound. Am J Obstet Gynecol 1970;106(2):279-85.

[3] Crawford JM. A study of human placental growth with observations on the placenta in erythroblastosis foetali. J Obstet Gynecol Br Emp 1959;66:885-96.

[4] Kohorn EI, Walker RHS, Morrison J, et al. Placental localization. Am J Obstet Gynecol 1969;103(6):868-77.

[5] Grannum PAT, Hobbins JC. The placenta. Radiol Clin North Am 1982;20(2):353-65.

[6] Hills D, Irwin GA, Tuck S, et al. Distribution of placental grades in high-risk gravidas. Am J Roentgenol 1984;143(5):1011-3.

[7] Daftary SN, Chakravarti S. Holland and Brews - Manual of obstetrics. $16^{\text {th }}$ edn. New Delhi: B.I. Churchill Livingstone Pvt. Ltd., 1998: p. 23-32.

[8] Hendricks CH. Patterns of foetal and placental growth: In the second half of normal pregnancy. Obstet Gynecol 1964;24(3):357-65.

[9] Granum PAT, Berkowitz RL, Hobbins JC. The ultrasonic changes in the maturing placenta and their relation to foetal pulmonic maturity. Am J Obstet Gynecol 1979;133(8):915-22.

[10] Bonds DR, Gabbe SG, Kumar S, et al. Fetal weight/ placental weight ratio and perinatal outcome. Am J Obstet Gynecol 1984;149(2):195-200.

[11] Bleker OP, Kloosterman GJ, Breur W, et al. The volumetric growth of human placenta: a longitudinal ultrasonic study. Am J Obstet Gynecol 1977;127(6):65761.

[12] Jauniaux E, Ramsay B, Campbell S. Ultrasonographic investigation of placental morphology and size during second trimester of pregnancy. Am J Obstet Gynecol 1994;170(1 Pt 1):130-7.

[13] Bovicelli L, Orsini LF, Rizzo N, et al. Estimation of gestational age during the first trimester by real-time measurement of foetal CRL and BPD. J Clin Ultrasound 1981;9(2):71-5.

[14] Wolf H, Oosting H, Treffers PE. A longitudinal study of relationship between placental and foetal growth as measured by ultrasonography. Am J Obstet Gynecol 1989;161(5):1140-5. 
[15] Mital P, Hooja N, Mehndiratta K. Placental thickness - a sonographic parameter for estimating gestational age of the foetus. Ind J Radiol Imag 2002;12(4):553-4.

[16] Anupama J, Ganesh K, Agarwal U, et al. Placental thickness - a sonographic indicator of gestational age. Journal of obstetrics and gynaecology of India 2001;51(3):48-9.
[17] Ohagwu CC, Abu PO, Udoh BE. Placental thickness: a sonographic indicator of gestational age in normal singleton pregnancies in Nigeria. Internet Journal of Medical Update 2009;4(2):9-14.

[18] Hoddick WK, Mahony BS, Callen FW, et al. Placental thickness. J Ultrasound Med 1985;4(9):479-82. 